\title{
Alkene Syn- and Anti-Oxyamination with Malonoyl Peroxides
}

\author{
Jonathan M. Curle, Marina C. Perieteanu, Philip G. Humphreys, Alan R. Kennedy,
} and Nicholas C. O. Tomkinson*

Cite This: https://dx.doi.org/10.1021/acs.orglett.0c00253

Read Online

ACCESS | 岁 Metrics \& More | 回 Article Recommendations | SI Supporting Information
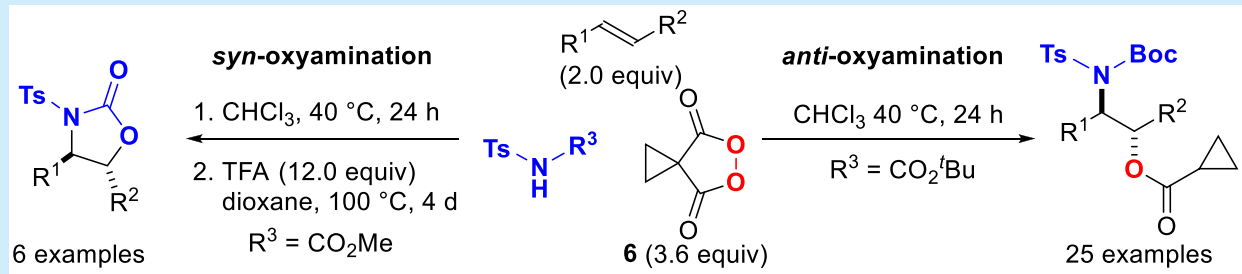

ABSTRACT: Malonoyl peroxide $\mathbf{6}$ is an effective reagent for the syn- or anti-oxyamination of alkenes. Reaction of $\mathbf{6}$ and an alkene in the presence of O-tert-butyl- $N$-tosylcarbamate $\left(\mathrm{R}^{3}=\mathrm{CO}_{2}{ }^{t} \mathrm{Bu}\right)$ leads to the anti-oxyaminated product in up to $99 \%$ yield. Use of $\mathrm{O}$ methyl- $N$-tosyl carbamate $\left(\mathrm{R}^{3}=\mathrm{CO}_{2} \mathrm{Me}\right)$ as the nitrogen nucleophile followed by treatment of the product with trifluoroacetic acid leads to the syn-oxyaminated product in up to $77 \%$ yield. Mechanisms consistent with the observed selectivities are proposed.

$\mathrm{T}$ he $\beta$-amino alcohol functionality is an important motif present in natural products, agrochemicals, pharmaceuticals, and ligands for catalysis. Many methods exist for the introduction of this functionality with the difunctionalization of alkenes representing a particularly efficient process. ${ }^{1}$ In the reaction of an alkene 1 , the oxyamination presents significant challenges with regard to regioselectivity and stereoselectivity with up to four possible products 2-5 (Scheme 1).

Scheme 1. Challenges of Oxyamination
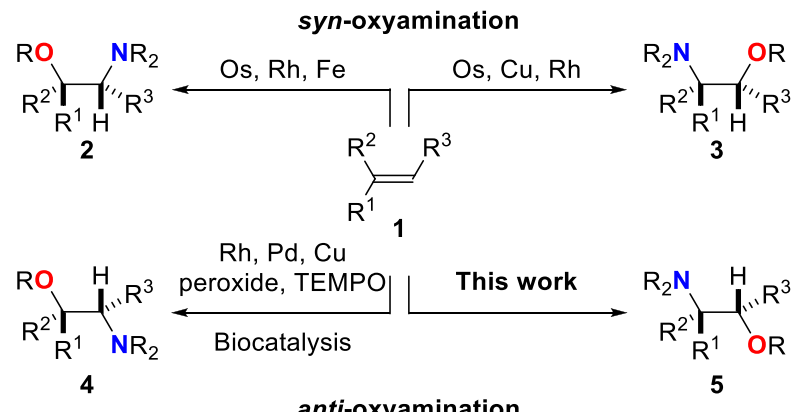

anti-oxyamination

Considerable attention has been devoted to the intramolecular (tethered) oxyamination of alkenes, which can circumvent regiochemistry issues; ${ }^{2}$ however, there are substantially fewer reports of intermolecular procedures which meet the regioand diastereochemical challenges of the process. ${ }^{3}$

For the preparation of the syn-products 2 and 3 through an intermolecular oxyamination the osmium catalyzed asymmetric aminohydroxylation developed by Sharpless represents the gold standard within the field. ${ }^{1,4}$ Loss of selectivity for some alkene substrates along with deficiencies in regioselectivity and the desire to prepare the anti-products 4 and 5 have driven further investigation. Important advances have been made with a variety of transition metals including osmium, ${ }^{5}$ rhodium, ${ }^{6}$ palladium, ${ }^{7}$ copper, ${ }^{8}$ and iron. ${ }^{9}$ Metal-free methods for the intermolecular oxyamination of alkenes have also been developed which include the use of TEMPO ${ }^{10}$ or peroxides. $^{11}$ In addition, Arnold reported a biocatalytic method for antioxyamination using a hemoprotein. ${ }^{12}$ While these recent developments represent excellent progress, diastereoselectivity in the majority of these transformations is not well explored and provides the impetus for additional research efforts. It is also noteworthy that stereoselective intermolecular methods to access anti-oxyamination product 5 are particularly limited. ${ }^{13}$ Within this manuscript, we report the development of an intermolecular metal-free anti-oxyamination through the reaction of an alkene 1 , malonoyl peroxide 6 and a nitrogen nucleophile and show how the product can be converted directly into the syn-oxyaminated product by treatment with TFA.

The investigation began with the reaction of trans-stilbene 7 and malonoyl peroxide $\mathbf{6}^{14,15}$ in the presence of different nitrogen nucleophiles. The aim was to find a nitrogen nucleophile that reacted with dioxonium $\mathbf{8}$ and not peroxide 6. ${ }^{16}$ From a total of 12 nitrogen nucleophiles examined, only saccharin 10 showed the desired activity (see the Supporting

Received: January 17, 2020 
Information for full details of screen). Reaction of alkene 7 (1.0 equiv), peroxide 6 ( 1.8 equiv), and saccharin 10 (2.0 equiv) in chloroform at $40{ }^{\circ} \mathrm{C}$ for $24 \mathrm{~h}$ gave the antioxyaminated product 11 (30\%) (Scheme 2). Saccharin 10 is an

Scheme 2. Alkene Oxyamination in the Presence of Saccharin

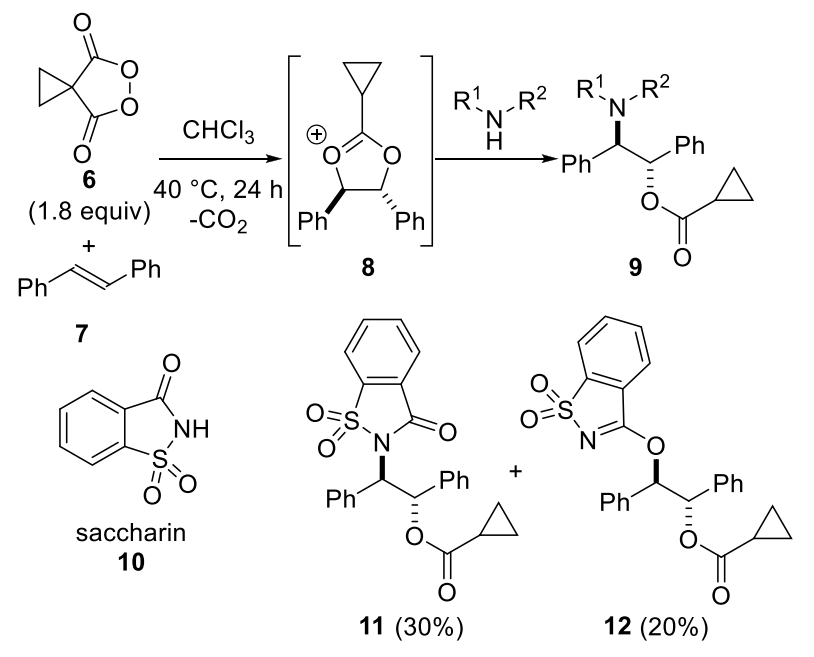

ambident nucleophile which can react through either its nitrogen or oxygen atom. ${ }^{17}$ Along with the oxyaminated product 11 the anti-dioxygenated coproduct 12 was also isolated from the reaction mixture in $20 \%$ yield. The structures of both 11 and 12 were confirmed by single-crystal X-ray crystallography (see the Supporting Information for full details). In contrast to the related intramolecular oxyamination procedure, $^{2 \mathrm{~b}}$ the product isolated has undergone decarboxylation. It is proposed that the low nucleophilicity of the amine nucleophile allows for decaboxylation of the initial adduct ${ }^{16}$ to give 8 prior to trapping with saccharin. We believed synthesis of 11 represented a simple and effective anti-oxyamination which proceeded under mild conditions and warranted further investigation.

We sought to understand the ambident reactivity of saccharin 10 to improve the selectivity for $N$-alkylation over $\mathrm{O}$-alkylation. Literature reports suggest the reactivity of ambident nucleophiles can be altered through changes in solvent and temperature; ${ }^{18}$ however, despite extensive investigation we were unable to significantly alter the ratio of 11 and 12 obtained. We therefore turned our attention to modifying the structure of saccharin 10. Seven acyl sulfonamide derivatives 13-19 were prepared by altering both the steric and electronic environments of the nitrogen atom, which were then reacted with stilbene 7 in the presence of malonoyl peroxide $6\left(\mathrm{CHCl}_{3}, 40{ }^{\circ} \mathrm{C}, 24 \mathrm{~h}\right)$ (Table 1$) . \mathrm{N}$ (Methylsulfonyl)acetamide 13 represents the simplest nucleophile to contain the acyl sulfonamide moiety and was found to produce the oxyaminated product 20A and dioxygenated coproduct $20 \mathrm{~B}$ in a combined yield of $38 \%$ as a $1: 1$ mixture (entry 1). Nucleophiles 14, 15, and 16 were prepared to study the influence of the steric environment around the heteroatoms on the transformation. Under the reaction conditions examined, all three examples were selective toward $O$-alkylation, resulting in the anti-dioxygenated products $21 \mathbf{B}-$ 23B (entries 2-4; 22-60\%). The added steric bulk clearly shielded the nitrogen atom, leading to the observed $O$ -
Table 1. Optimization of Nitrogen Nucleophile

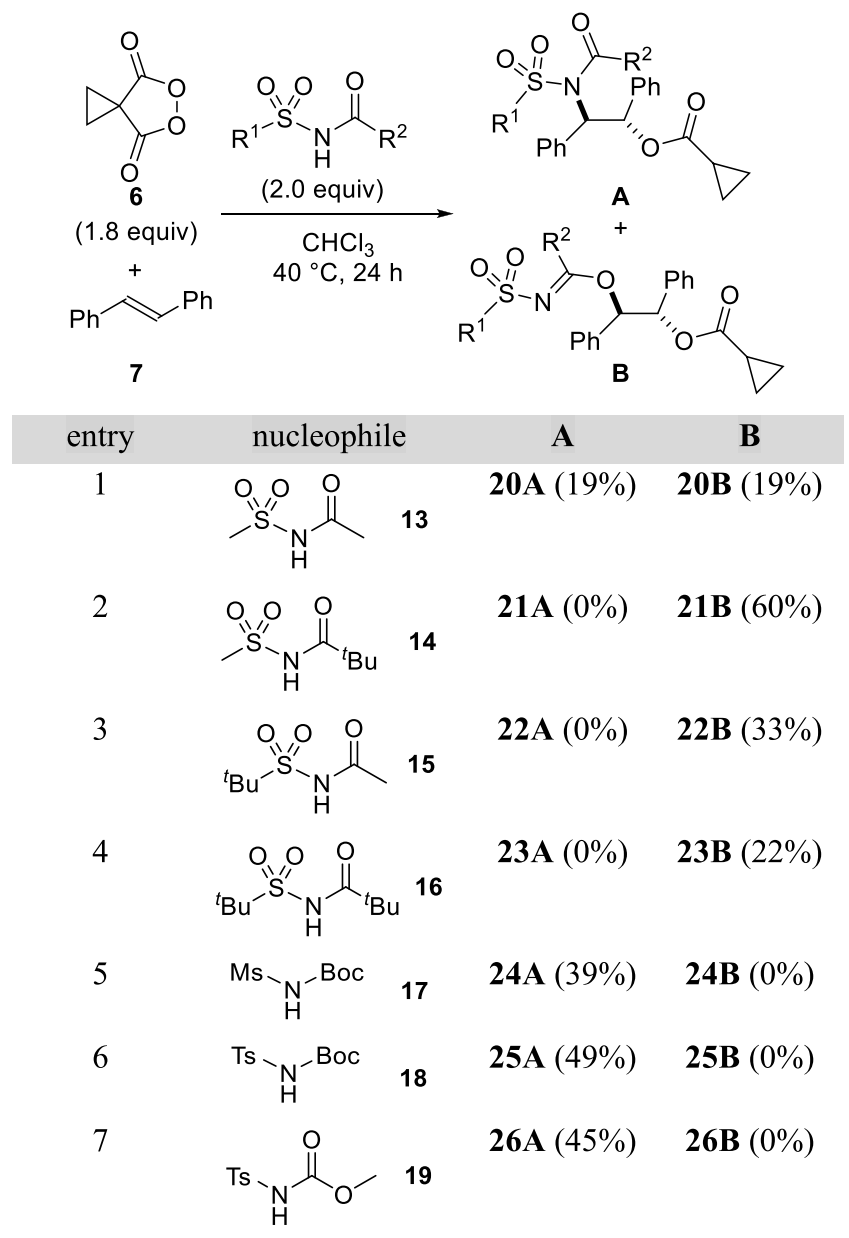

selectivity. We therefore altered the electronic environment of the nitrogen nucleophile by preparing the $N$-sulfonyl carbamates 17-19. Under standard reaction conditions, all three nucleophiles were $N$-selective, providing the antioxyaminated products 24A-26A (entries 5-7; 39-49\%). This remarkable switch in selectivity by changing the steric or electronic environment of the nitrogen nucleophile represents a powerful observation that presents an intriguing opportunity for further investigation.

$O$-tert-Butyl- $N$-tosylcarbamate $\mathbf{1 8}$ was selected as the preferred nucleophile. Further optimization of the reaction conditions failed to improve the yield of oxyamination product 25A beyond 49\%. However, the conversion of nucleophile 18 to oxyaminated product $25 \mathrm{~A}$ was an efficient process. Therefore, in examining the substrate scope of the reaction we employed the conditions outlined in Scheme 3 (entry 1, $97 \%)$, using the nitrogen nucleophile $\mathbf{1 8}$ as the limiting reagent.

Examination of a series of stilbene derivatives showed the reaction to proceed efficiently at room temperature with complete anti-diastereoselectivity (Scheme 3 ). The reaction was tolerant of substitution in the 2-, 3-, and 4-positions of the stilbene substrate (entries 2-4, 62-92\%). In addition, fluorine (entry 6, $71 \%$ ), chlorine (entry $7,90 \%$ ), and bromine (entry 8 , $85 \%$ ) substituents on the aromatic ring also led to the expected products, providing useful handles for further synthetic manipulation. Alternative $\mathrm{N}$ - and $\mathrm{O}$-substituted carbamates 
Scheme 3. Stilbene Substrate Scope ${ }^{a}$

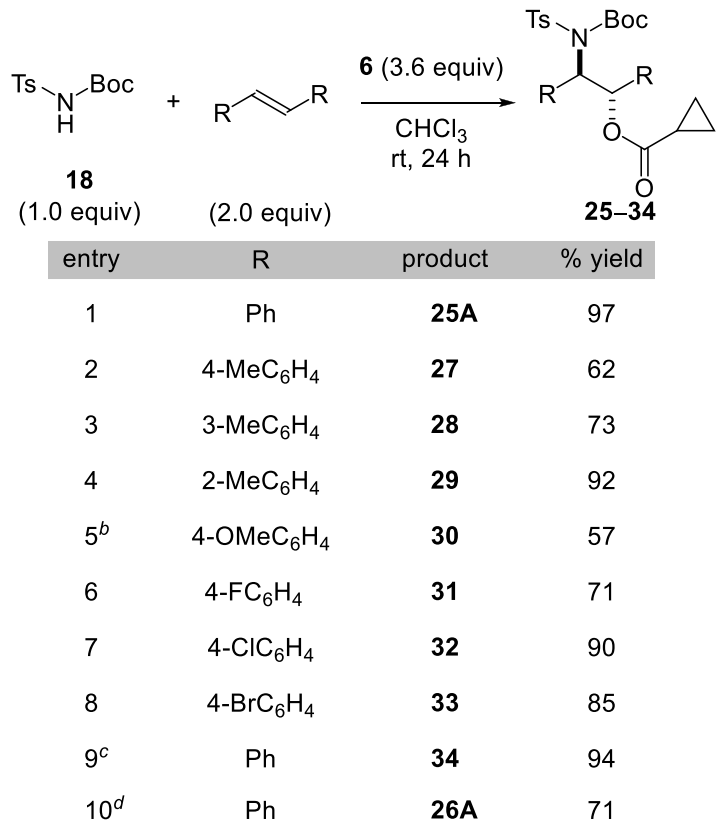

${ }^{a}$ All reactions conducted in duplicate. ${ }^{b}$ Reaction conducted at $0{ }^{\circ} \mathrm{C}$. ${ }^{c} \mathrm{O}$-tert-Butyl- $\mathrm{N}$-((4-cyanophenyl)sulfonyl)carbamate 35 was used as nucleophile. ${ }^{d} \mathrm{O}$-Methyl- $N$-tosylcarbamate 19 was used as nucleophile.

were also tolerated under the optimized reaction conditions. For example, O-tert-butyl- $N$-((4-cyanophenyl)sulfonyl)carbamate 35 (entry 9, 94\%) and $O$-methyl- $N$-tosylcarbamate 19 (entry 10, 71\%) both gave the expected anti-oxyaminated products in excellent yields.

Our attention then turned to styrene substrates (Scheme 4). Reaction of styrene, peroxide 6 , and amine nucleophile 18 $\left(\mathrm{CHCl}_{3}, 40{ }^{\circ} \mathrm{C}, 24 \mathrm{~h}\right)$ gave oxyaminated product $37 \mathrm{~A}$ along with the regioisomer $37 \mathbf{B}$ in a 3.5:1 ratio (Scheme 4, entry 1 ; $77 \%$ ). The expected product $37 \mathrm{~A}$ is a result of the nucleophile 18 adding to the benzylic position $\mathbf{A}$ of dioxonium intermediate 36. The minor regioisomer $37 \mathbf{B}$ arises through addition of $\mathbf{1 8}$ to the more sterically accessible position $\mathbf{B}$. The amount of the major regioisomer $\mathbf{A}$ can be increased by the introduction of electron-donating substituents to the aromatic ring. For example, a methyl group can increase the amount of the major isomer to up to $10: 1$ (Scheme 4, entries 2-4; 76$99 \%)$. This ratio increases further using mesityl styrene as the substrate (entry 5, 20:1; 78\%). 4-Methoxystyrene provides the expected oxyaminated product $\mathbf{4 2}$ with complete selectivity for addition of the nucleophile at position A (entry 6, 47\%). Using halogen-substituted styrenes lowers the ratio of products $\mathbf{A} / \mathbf{B}$ observed as the substituent is moved from para (entries 1012 , up to $5: 1$ ) to meta (entry $8,1.4: 1$ ) to ortho positions (entry $7,1: 1)$. We believe selectivity and reactivity are altered by a combination of lone pair stabilization and the electronwithdrawing nature of the substituents destabilizing any buildup of positive charge at position $\mathbf{A}$ of proposed intermediate 36. Introducing substitution at the $\beta$-position of the styrene substrate results in complete stereoselectivity in the oxyamination process for addition of the nucleophile at position A (Scheme 4, entries 13-15; 81-92\%). This steric factor completely overrides any electronic influence on the regiochemical outcome of the transformation (cf. entries 7 vs 14). The reaction of cis- $\beta$-methylstyrene proceeded with
Scheme 4. Styrene Substrate Scope ${ }^{a}$

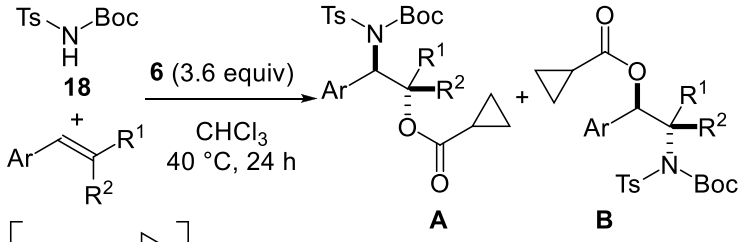

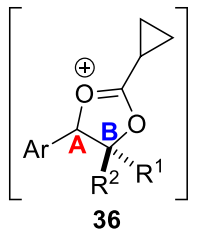<smiles>[R]=CCC(=O)C1CC1</smiles><smiles></smiles><smiles>[R10]CC(c1ccccc1)N(C)O</smiles>

37A:37B 3.5:1 (77\%)

2.<smiles>[R7]CC(c1ccc(C)cc1)N([13CH3])C(=O)OC(C)(C)C</smiles>

3. $\quad \mathrm{Ts}_{-N^{-}}$- Boc

4. $\mathrm{Ts}_{-\mathrm{N}^{-}}-\mathrm{BOC}$

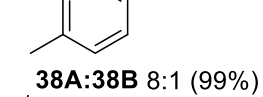<smiles>[R]OCC(C)c1cccc(C)c1</smiles>

$5 . b$
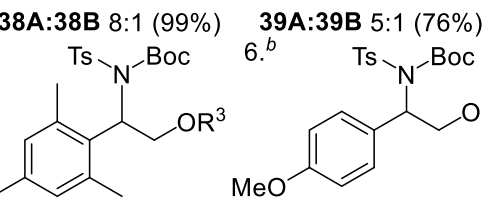<smiles>[R]OCC(c1ccc(OC)cc1)N([13CH3])C(=O)OCc1ccccc1</smiles>

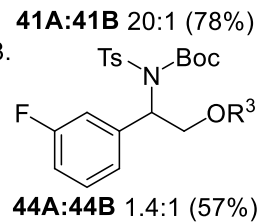

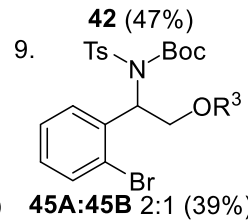<smiles>Cc1ccccc1C(C)CO[Ga]</smiles>

40A:40B 10:1 (83\%)

7. Ts ${ }_{-}-B O C$<smiles>[R]OCC(N)c1ccccc1F</smiles>

43A:43B 1:1 (65\%)

10. $\mathrm{Ts}_{-N^{-}}-\mathrm{BOC}$

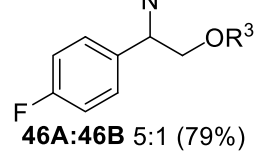

11. Ts $\backslash_{N^{-}}-\mathrm{Boc}$<smiles>[R1]OCC(N)c1ccc(Cl)cc1</smiles>

Ts $\mathrm{BOC}$

13. Ts $\backslash_{-}-\mathrm{Boc}$<smiles>[R20]C(C)C(c1ccccc1)N([13CH3])[13CH3]</smiles>

14
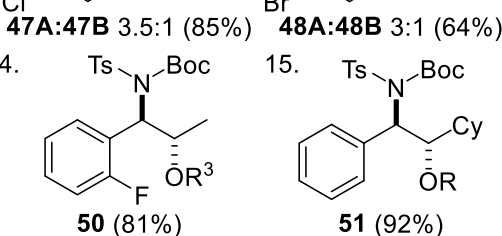

15. Ts

16. ${ }^{c}$ Ts - - Boc<smiles>[R10]C(C)C(c1ccccc1)N([13CH3])C(=O)OC(C)(C)C</smiles>

52 syn:anti 1.4:1 (54\%)

${ }^{a}$ All reactions were conducted in duplicate, with combined yield of regioisomers quoted. ${ }^{b}$ Reaction conducted at $25{ }^{\circ} \mathrm{C} .{ }^{c}$ cis- $\beta$ methylstyrene was used as the alkene substrate.

complete regioselectivity; however, considerable loss in stereoselectivity was observed, suggesting that cis-alkenes will be poor substrates within this transformation (entry 16).

Reaction of $O$-methyl- $N$-tosylcarbamate 19 with stilbene 7 and malonoyl peroxide 6 under the standard reaction conditions provided the oxyaminated product 26A (Scheme 3 , entry $10,71 \%)$. Treatment of this adduct with trifluoroacetic acid (12 equiv) in $\mathrm{CH}_{2} \mathrm{Cl}_{2}\left(40{ }^{\circ} \mathrm{C}, 5 \mathrm{~h}\right)$ led to the oxazolidinone 53 ( $77 \%$ over two steps), the product of a formal syn-oxyamination of the trans-stilbene substrate 7 (Scheme 5 , entry 1 ). This provides a powerful and particularly useful complementary strategy to the anti-oxyamination procedure described above, allowing access to both diastereomeric oxyaminated products using the same alkene and malonoyl peroxide reagents. This strategy was also effective for styrene (entry 2) and $\beta$-substituted styrene derivatives (entries 3 and 4). Consistent with previous observations, 2fluorostyrene provided the two regioisomeric products $\mathbf{5 7}$ and 58 after oxazolidinone formation (entry 5, 72\%), the structures of which were confirmed by X-ray crystallography (see the 


\section{Scheme 5. Anti-Oxymaination of Alkenes}

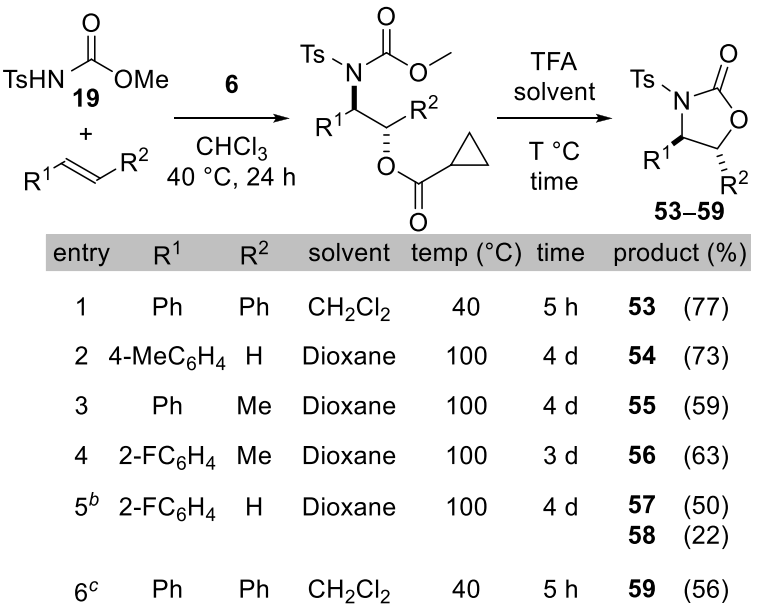

${ }^{a}$ All reactions conducted in duplicate. ${ }^{b} 58$ corresponds to the regioisomer. ${ }^{c}$ Methyl ((4-cyanophenyl)sulfonyl)carbamate 60 used as nucleophile.

Supporting Information for full details). The alternative nitrogen nucleophile $\mathrm{O}$-methyl- $\mathrm{N}$-((4-cyanophenyl)sulfonyl)carbamate 60 could also be used effectively within this synthetic sequence (entry 6, 56\%).

A mechanism consistent with the observed selectivities is outlined in Scheme 6. Reaction of malonoyl peroxide 6 with

Scheme 6. Proposed Mechanism for the Anti- and SynOxyamination
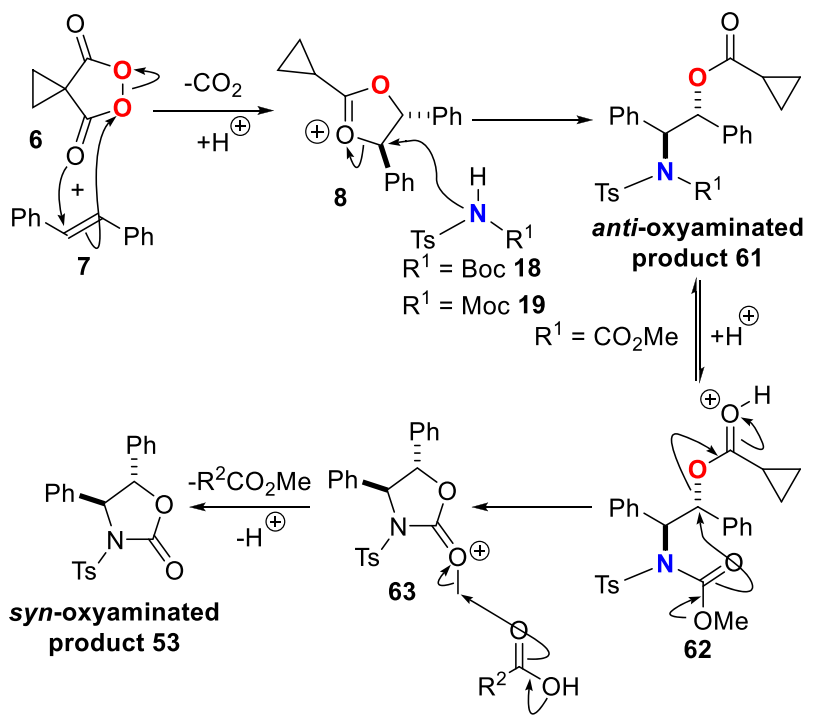

the alkene leads to the syn-dioxonium intermediate $\mathbf{8}^{16}$ Interception of $\mathbf{8}$ with the weak nitrogen nucleophile $\mathbf{1 8}$ (or 19) via an $S_{N} 2$ process leads to the anti-oxyaminated product 61 which can be isolated and purified. Subsequent reaction of $61\left(\mathrm{R}^{1}=\mathrm{CO}_{2} \mathrm{Me}\right)$ under acidic conditions gives 62 , which can undergo a 5-exo-tet cyclization, inverting the relative stereochemistry of the oxygen substituent and leading to 63 . Reaction of intermediate $\mathbf{6 3}$ with either trifluoroacetic acid or cyclopropane carboxylic acid gives the syn-oxyaminated product 53. ${ }^{19}$ Doping experiments confirmed the presence of both methyl trifluoroacetate and methyl cyclopropane carboxylate within the crude reaction mixture (see the
Supporting Information for full details) consistent with this proposal.

Selective removal of the oxygen and nitrogen protecting groups on the oxyaminated products was possible (Scheme 7),

Scheme 7. Removal of Nitrogen and Oxygen Protecting Groups $^{a}$
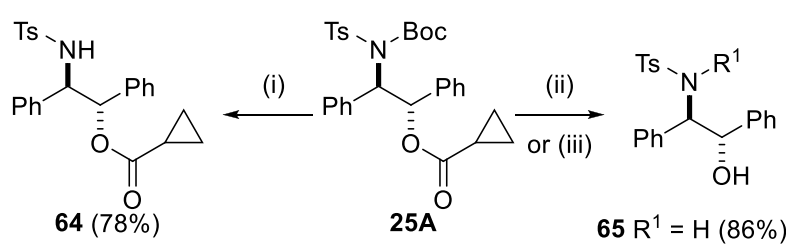

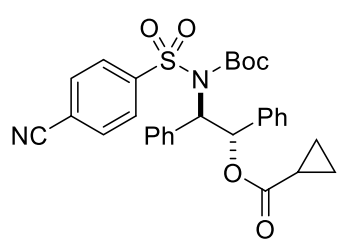

34

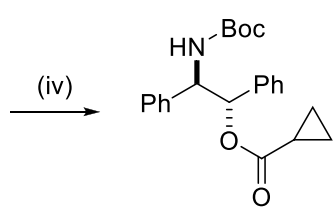

$67(73 \%)$
${ }^{a}$ Reagents and conditions: (i) HCI (4 equiv), dioxane, $60{ }^{\circ} \mathrm{C}, 8 \mathrm{~h}$; (ii) $\mathrm{MeNH}_{2}, \mathrm{EtOH}, 40{ }^{\circ} \mathrm{C}, 18 \mathrm{~h}$; (iii) $\mathrm{K}_{2} \mathrm{CO}_{3}$ (5 equiv), $\mathrm{MeOH} / \mathrm{CH}_{2} \mathrm{Cl}_{2}$ (1:1), rt, $18 \mathrm{~h}$; (iv) 1-dodecanethiol (5 equiv), DBU (4.8 equiv), DMF, rt, $5 \mathrm{~h}$.

providing the opportunity for further elaboration. Reaction of 25A with $\mathrm{HCl}$ (4 equiv) in dioxane at $60{ }^{\circ} \mathrm{C}$ selectively removed the Boc group (64,78\%). Treatment of $25 \mathrm{~A}$ with $\mathrm{K}_{2} \mathrm{CO}_{3}$ in methanol removed the ester protecting group (66, $72 \%)$, whereas both the ester and carbamate could be removed by reaction with methylamine in ethanol $(65,86 \%)$. In addition, the 4-cyanobenzenesulfonamide group could be removed selectively using 1-dodecanethiol and DBU in DMF to give $67(73 \%) .^{20}$ This sulfonamide protecting group could also be removed from the syn-oxyaminated product $\mathbf{5 9}(68 \%)$ (not shown, see the Supporting Information for full details).

In conclusion, we have shown that malonoyl peroxide $\mathbf{6}$ is an effective reagent for the intermolecular anti-oxyamination of a series of stilbene and styrene substrates in the presence of a nitrogen nucleophile. Optimization of the nitrogen nucleophile showed that $\mathrm{N}$-sulfonyl carbamates formed the new $\mathrm{C}-\mathrm{N}$ bond most efficiently. Stereoselectivity for the transformation was excellent with the reaction leading efficiently to the antioxyamination product. The regiochemcal outcome of the reaction is influenced by electronics, with the reaction of electron-rich alkenes proceeding with the highest regioselectivity; however, this subtle electronic influence is overridden by sterics. It also proved possible to convert the anti-oxyaminated product to the syn-diastereoisomer by treatment of the crude product with trifluoroacetic acid providing an effective method for the preparation of both the syn- and anti-oxyaminated product from reaction of the same alkene, nitrogen nucleophile, and peroxide. Effective methods for the selective or concomitant removal of substituents on both the nitrogen and oxygen atoms suggest this simple procedure, which extends recent advances in the chemistry of diacylperoxides, ${ }^{21}$ should find broad use within synthesis. 


\section{ASSOCIATED CONTENT}

\section{(s) Supporting Information}

The Supporting Information is available free of charge at https://pubs.acs.org/doi/10.1021/acs.orglett.0c00253.

Analytical data, experimental procedures, and NMR spectra for all compounds reported (PDF)

\section{Accession Codes}

CCDC 1951983-1951986 contain the supplementary crystallographic data for this paper. These data can be obtained free of charge via www.ccdc.cam.ac.uk/data_request/cif, or by emailing data_request@ccdc.cam.ac.uk, or by contacting The Cambridge Crystallographic Data Centre, 12 Union Road, Cambridge CB2 1EZ, UK; fax: +44 1223336033.

\section{AUTHOR INFORMATION}

\section{Corresponding Author}

Nicholas C. O. Tomkinson - Department of Pure and Applied Chemistry, WestCHEM, Thomas Graham Building, University of Strathclyde, Glasgow G1 1XL, U.K.; 잉o.org/00000002-5509-0133; Email: nicholas.tomkinson@strath.ac.uk

\section{Authors}

Jonathan M. Curle - Department of Pure and Applied Chemistry, WestCHEM, Thomas Graham Building, University of Strathclyde, Glasgow G1 1XL, U.K.

Marina C. Perieteanu - Department of Pure and Applied Chemistry, WestCHEM, Thomas Graham Building, University of Strathclyde, Glasgow G1 1XL, U.K.

Philip G. Humphreys - GlaxoSmithKline Medicines Research Centre, Stevenage SG1 2NY, U.K.; 이이.orid.org/0000-00028614-7155

Alan R. Kennedy - Department of Pure and Applied Chemistry, WestCHEM, Thomas Graham Building, University of Strathclyde, Glasgow G1 1XL, U.K.; O orcid.org/0000-00033652-6015

Complete contact information is available at:

https://pubs.acs.org/10.1021/acs.orglett.0c00253

\section{Notes}

The authors declare no competing financial interest.

\section{ACKNOWLEDGMENTS}

We thank the EPSRC for funding via Prosperity Partnership EP/S035990/1 and the University of Strathclyde and GlaxoSmithKline for financial support. We also thank the EPSRC Mass Spectrometry Service, Swansea, for highresolution spectra.

\section{REFERENCES}

(1) For reviews of oxyamination, see: (a) O'Brien, P. Sharpless Asymmetric Aminohydroxylation: Scope, Limitations, and Use in Synthesis. Angew. Chem., Int. Ed. 1999, 38, 326-329. (b) Nilov, D.; Reiser, O. The Sharpless Asymmetric Aminohydroxylation - Scope and Limitation. Adv. Synth. Catal. 2002, 344, 1169-1173. (c) Bodkin, J. A.; McLeod, M. D. The Sharpless asymmetric aminohydroxylation. J. Chem. Soc., Perkin Trans. 2002, 1, 2733-2746. (d) Donohoe, T. J.; Callens, C. K. A.; Flores, A.; Lacy, A. R.; Rathi, A. H. Recent Developments in Methodology for the Direct Oxyamination of Olefins. Chem. - Eur. J. 2011, 17, 58-76. (e) Donohoe, T. J.; Callens, C. K. A.; Lacy, A. R.; Winter, C. Tethered Aminohydroxylation
Reaction and Its Application to Total Synthesis. Eur. J. Org. Chem. 2012, 2012, 655-663.

(2) For selected recent advances in intramolecular (tethered) oxyamination processes, see: (a) Reed, N. L.; Herman, M. I.; Miltchev, V. P.; Yoon, T. P. Photocatalytic Oxyamination of Alkenes: Copper(II) Salts as Terminal Oxidants in Photoredox Catalysis. Org. Lett. 2018, 20, 7345-7350. (b) Alamillo-Ferrer, C.; Curle, J. M.; Davidson, S. C.; Lucas, S. C. C.; Atkinson, S. J.; Campbell, M.; Kennedy, A. R.; Tomkinson, N. C. O. Alkene Oxyamination Using Malonoyl Peroxides: Preparation of Pyrrolidines and Isoxazolidines. J. Org. Chem. 2018, 83, 6728-6740. (c) Feige, P.; de Haro, T.; Rusconi, G.; Merino, E.; Nevado, C. Gold-Catalyzed Oxidative Aminoesterification of Unactivated Alkenes. Monatsh. Chem. 2018, 149, 749-754. (d) Wu, F.; Stewart, S.; Ariyarathna, J. P.; Li, W. Aerobic Copper Catalyzed Alkene Oxyamination for Amino Lactone Synthesis. ACS Catal. 2018, 8, 1921-1925. (e) Liu, R.-H.; Wang, Z.-Q.; Wei, B.-Y.; Zhang, J.-W.; Zhou, B.; Han, B. Cu-Catalyzed Aminoacyloxylation of Unactivated Alkenes of Unsaturated Hydrazones with Manifold Carboxylic Acids toward Ester-Functionalized Pyrazolines. Org. Lett. 2018, 20, 4183-4186. (f) Manick, A.-D.; Aubert, S.; Yalcouye, B.; Prange, T.; Berhal, F.; Prestat, G. Access to Functionalized Imidazolidin-2-one Derivatives by Iron-Catalyzed Oxyamination of Alkenes. Chem. - Eur. J. 2018, 24, 11485-11492. (g) Hemric, B. N.; Chen, A. W.; Wang, Q. Copper-Catalyzed Modular Amino Oxygenation of Alkenes: Access to Diverse 1,2Amino Oxygen-Containing Skeletons. J. Org. Chem. 2019, 84, 14681488.

(3) For related methods for the azidohydroxylation of alkenes, see: (a) Zhang, B.; Studer, A. Stereoselective Radical Azidooxygenation of Alkenes. Org. Lett. 2013, 15, 4548-4551. (b) Prasad, P. K.; Reddi, R. N.; Sudalai, A. Oxidant Controlled Regio- and Stereodivergent Azidohydroxylation of Alkenes via $\mathrm{I}_{2}$ Catalysis. Chem. Commun. 2015, 51, 10276-10279. (c) Sun, X.; Li, X.; Song, S.; Zhu, Y.; Liang, Y.-F.; Jiao, N. Mn-Catalyzed Highly Efficient Aerobic Oxidative Hydroxyazidation of Olefins: A Direct Approach to $\beta$-Azido Alcohols. J. Am. Chem. Soc. 2015, 137, 6059-6066. (d) Yang, B.; Lu, Z. Visible-LightPromoted Metal-Free Aerobic Hydroxyazidation of Alkenes. ACS Catal. 2017, 7, 8362-8365.

(4) Li, G.; Chang, H.-T.; Sharpless, K. B. Catalytic Asymmetric Aminohydroxylation (AA) of Olefins. Angew. Chem., Int. Ed. Engl. 1996, 35, 451-454.

(5) (a) Masruri; Willis, A. C.; McLeod, M. D. Osmium-Catalyzed Vicinal Oxyamination of Alkenes by $\mathrm{N}$-(4-Toluenesulfonyloxy)carbamates. J. Org. Chem. 2012, 77, 8480-8491. (b) Ma, Z.; Naylor, B. C.; Loertscher, B. M.; Hafen, D. D.; Li, J. M.; Castle, S. L. Regioselective Base-Free Intermolecular Aminohydroxylations of Hindered and Functionalized Alkenes. J. Org. Chem. 2012, 77, $1208-1214$

(6) (a) Beaumont, S.; Pons, V.; Retailleau, P.; Dodd, R. H.; Dauban, P. Catalytic Oxyamidation of Indoles. Angew. Chem., Int. Ed. 2010, 49, 1634-1637. (b) Ciesielski, J.; Dequirez, G.; Retailleau, P.; Gandon, V.; Dauban, P. Rhodium-Catalyzed Alkene Difunctionalization with Nitrenes. Chem. - Eur. J. 2016, 22, 9338-9347. (c) Shi, Y.; Wang, Y.; Lu, X.; Zhang, Y.; Wu, Y.; Zhong, F. Rhodium-Catalyzed Aminohydroxylation of Unactivated Alkenes in Aqueous Media for the Benign Synthesis of 1,2-Amino Alcohols. Green Chem. 2019, 21, 780784.

(7) Liu, G.; Stahl, S. S. Highly Regioselective Pd-Catalyzed Intermolecular Aminoacetoxylation of Alkenes and Evidence for cisAminopalladation and $\mathrm{S}_{\mathrm{N}} 2 \mathrm{C}-\mathrm{O}$ Bond Formation. J. Am. Chem. Soc. 2006, 128, 7179-7181.

(8) (a) Michaelis, D. J.; Shaffer, C. J.; Yoon, T. P. Copper(II)Catalyzed Aminohydroxylation of Olefins. J. Am. Chem. Soc. 2007, 129, 1866-1867. (b) Hemric, B. N.; Wang, Q. Copper-Catalyzed Intermolecular Oxyamination of Olefins using Carboxylic Acids and O-Benzoylhydroxylamines. Beilstein J. Org. Chem. 2016, 12, 22-28. (c) Herrera-Leyton, C.; Madrid-Rojas, M.; Lopez, J. J.; Canete, A.; Hermosilla-Ibanez, P.; Perez, E. G. Copper-Catalyzed Intermolecular 
Aminooxygenation of Styrenes using $N$-Fluorobenzenesulfonimide and Simple Alcohols. ChemCatChem 2016, 8, 2015-2018.

(9) Lu, D.-F.; Zhu, C.-L.; Jia, Z.-X.; Xu, H. Iron(II)-Catalyzed Intermolecular Amino-Oxygenation of Olefins through the $\mathrm{N}-\mathrm{O}$ Bond Cleavage of Functionalized Hydroxylamines. J. Am. Chem. Soc. 2014, 136, 13186-13189.

(10) Weng, S.-S.; Zhang, J.-W. N-Oxyl-Radical-Catalyzed Intermolecular Aminooxygenation of Styrenes and Inter/intramolecular Aminoalkoxylation of Homoallylic Alcohols. ChemCatChem 2016, 8, $3720-3724$.

(11) Xue, Q.; Xie, J.; Xu, P.; Hu, K.; Cheng, Y.; Zhu, C. Metal-Free, $\mathrm{n}-\mathrm{Bu}_{4} \mathrm{NI}$-Catalyzed Regioselective Difunctionalization of Unactivated Alkenes. ACS Catal. 2013, 3, 1365-1368.

(12) Cho, I.; Prier, C. K.; Jia, Z.-J.; Zhang, R. K.; Görbe, T.; Arnold, F. H. Enantioselective Aminohydroxylation of Styrenyl Olefins Catalyzed by an Engineered Hemoprotein. Angew. Chem., Int. Ed. 2019, 58, 3138-3142.

(13) Ren, S.; Song, S.; Ye, L.; Feng, C.; Loh, T.-P. Copper-catalyzed oxyamination of electron deficient alkenes with $\mathrm{N}$-acyloxyamines. Chem. Commun. 2016, 52, 10373-10376.

(14) For the syn-dihydroxylation of alkenes using peroxide 6, see: (a) Griffith, J. C.; Jones, K. M.; Picon, S.; Rawling, M. J.; Kariuki, B. M.; Campbell, M.; Tomkinson, N. C. O. Alkene Syn Dihydroxylation with Malonoyl Peroxides. J. Am. Chem. Soc. 2010, 132, 14409-14411. (b) Jones, K. M.; Tomkinson, N. C. O. Metal-Free Dihydroxylation of Alkenes using Cyclobutane Malonoyl Peroxide. J. Org. Chem. 2012, 77, 921-928. (c) Picon, S.; Rawling, M.; Campbell, M.; Tomkinson, N. C. O. Alkene Dihydroxylation with Malonoyl Peroxides: Catalysis Using Fluorinated Alcohols. Org. Lett. 2012, 14, 6250-6253.

(15) For the anti-dihydroxylation of alkenes using peroxide 6, see: (a) Alamillo-Ferrer, C.; Davidson, S. C.; Rawling, M. J.; Theodoulou, N. H.; Campbell, M.; Humphreys, P. G.; Kennedy, A. R.; Tomkinson, N. C. O. Alkene anti-Dihydroxylation with Malonoyl Peroxides. Org. Lett. 2015, 17, 5132-5135. (b) Alamillo-Ferrer, C.; KarabourniotisSotti, M.; Kennedy, A. R.; Campbell, M.; Tomkinson, N. C. O. Alkene Dioxygenation with Malonoyl Peroxides: Synthesis of $\gamma$-Lactones, Isobenzofuranones, and Tetrahydrofurans. Org. Lett. 2016, 18, 31023105.

(16) For mechanistic studies on the formation of dioxonium 8, see: Rawling, M. J.; Rowley, J. H.; Campbell, M.; Kennedy, A. R.; Parkinson, J. A.; Tomkinson, N. C. O. Mechanistic Insights into the Malonoyl Peroxide syn-Dihydroxylation of Alkenes. Chem. Sci. 2014, 5, 1777-1785.

(17) Greenberg, F. H. Saccharin Alkylation. J. Chem. Educ. 1990, 67, 611.

(18) (a) Torhan, M. C.; Peet, N. P.; Williams, J. D. A Comparison of $\mathrm{N}$ - versus $\mathrm{O}$-Alkylation of Substituted 2-Pyridones under Mitsunobu Conditions. Tetrahedron Lett. 2013, 54, 3926-3928. (b) Guo, Z.-X.; Cammidge, A. N.; McKillop, A.; Horwell, D. C. N-vs O-Alkylation in 2(1H)-Quinolinone Derivatives. Tetrahedron Lett. 1999, 40, 69997002. (c) Chung, N. M.; Tieckelmann, H. Alkylations of Heterocyclic Ambident Anions. IV. Alkylation of 5-Carbethoxy- and 5-Nitro-2Pyridone Salts. J. Org. Chem. 1970, 35, 2517-2520.

(19) For a related transformation, see: Kuszpit, M. R.; Giletto, M. B.; Jones, C. L.; Bethel, T. K.; Tepe, J. J. Hydroxyamination of Olefins Using Br-N-( $\left.\mathrm{CO}_{2} \mathrm{Me}\right)_{2}$. J. Org. Chem. 2015, 80, 1440-1445.

(20) Schmidt, M. A.; Stokes, R. W.; Davies, M. L.; Roberts, F. 4Cyanobenzenesulfonamides: Amine Synthesis and Protecting Strategy To Compliment the Nosyl Group. J. Org. Chem. 2017, 82, 45504560.

(21) For selected recent examples, see: (a) Pilevar, A.; Hosseini, A.; Becker, J.; Schreiner, P. R. Syn-Dihydroxylation of Alkenes Using a Sterically Demanding Cyclic Diacyl Peroxide. J. Org. Chem. 2019, 84, 12377-12386. (b) Vil', V. A.; Gorlov, E. S.; Bityukov, O. V.; Barsegyan, Y. A.; Romanova, Y. E.; Merkulova, V. M.; Trente'ev, A. O. Adv. Synth. Catal. 2019, 361, 3173-3181. (c) Pilevar, A.; Hosseini, A.; Šekutor, M.; Hausmann, H.; Becker, J.; Turke, K.; Schreiner, P. R. Tuning the Reactivity of Peroxo Anhydrides for Aromatic C-H Bond Oxidation. J. Org. Chem. 2018, 83, 10070-10079. 